\title{
Investigation of biomechanical parameters of walking in the exoskeleton in patients with complete lower palsy
}

\begin{abstract}
Method of investigation of patients with complete lower palsy, who could walk only in the exoskeleton, has been proposed in this work. Application of the force measure insoles is the best method of investigation of walking of such patients. Investigation of a number of the ground reaction forces permits to carry out the detailed processing of the results, to analyze changes of stability and supportability in the process of adaptation of patient to walking in the exoskeleton, to retrace influence of combination of training in the exoskeleton with the other methods of rehabilitation. Parameters of walking in the exoskeleton «ExoAtlet» of patients with consequences of the spinal cord injury have been compared with the same parameters of walking of the healthy people.
\end{abstract}

Keywords: gait symmetry, locomotor cycle, SCI rehabilitation, exoskeleton, ground reaction force
Volume 4 Issue 3 - 2018

\author{
Pismennaya EV,' Petrushanskaya KA, ${ }^{2}$ \\ Shapkova EV ${ }^{3}$ \\ 'Institute of Mechanics of Moscow State University, Russia \\ ${ }^{2}$ ExoAtlet LLC, Russia \\ ${ }^{3}$ Saint-Petersburg State Research Institute of \\ Phthisiopulmonology, Russia
}

\begin{abstract}
Correspondence: Pismennaya EV, University Institute of Mechanics of Moscow State University, "ExoAtlet”, SanktPetersberg institute of phtisiopulmonology, Ist Truzhennikov lane, I5, Moscow, Russia, I I9121, Tel +7 (495) 374-85-30, Email info@exoatlet.ru, epismen@yandex.ru
\end{abstract}

Received: June 01, 2017 | Published: June 12, 2018

\section{Introduction}

Development of exoskeleton as assistive and rehabilitation equipment for people with the locomotor disturbances has become a world tendency.,2-8 Application of exoskeletons in patients with locomotor disturbances demands instrumental estimation of walking parameters. A number of the necessary primary parameters of walking and corresponding methods of investigations have been formed in the world biomechanics, namely, biomechanical, energetic and electromyographic. The following methods belong to the biomechanical ones: podometry, goniometry, and dynamometry, which record correspondingly the temporal, kinematic and dynamic parameters of walking. Investigation of the energetic parameters of walking supposes estimation of the energy expenditures by means of a method of mathematic modeling. At last, investigation of the electromyographic parameters supposes recording the electrical activity of muscles during the locomotor cycle, so called EMGpattern of muscles. ${ }^{9}$

But all these methods of investigations may be applied only in those patients, who can walk either independently or with the help of the corresponding person or with means of the additional support crutches, canes and walkers, but it is difficult to apply these methods in patients with complete lower palsy, walking in the exoskeleton. From our point of view, it is necessary to carry out not only clinical, but also instrumental investigations of walking even in such patients. All of the above-mentioned traditional methods are practically unfeasible in patients, walking in the exoskeleton. The following requirements are the main ones for investigation of patients, who can't walk independently-maximal information, but at the same time investigation must be unfatiguing. From our point of view, force measure insoles Tekscan completely satisfy these requirements. Application of the force measure insoles during walking in exoskeleton allows to receive the main, temporal and dynamic characteristics of walking in healthy examinees and in patients with palsies, to reveal similarity and distinctions between them. The main advantage of application of force measure insoles consists in possibility of recording the vertical component $\mathrm{Rz}$ of ground reaction force in each step, what gives an opportunity of receiving data of a number of steps. At the same time application of the tensometric platform permits to record the ground reaction force only of one step for the whole pass regardless of its length. Application of the force measure insoles saves time for investigations and permits to receive not only basic parameters of walking, but also their variability-an important parameter of mastering the skill of walking in the exoskeleton. ${ }^{10}$ The purpose of these investigations was to reveal the most informative parameters of estimation of walking in the exoskeleton.

\section{Material and methods of investigations}

With this purpose walking in the exoskeleton "ExoAtlet" has been investigated in 5 healthy men and in 6 patients with consequences of the spinal cord injury. Investigations have been carried out in SaintPetersburg State Research Institute of Phthisiopulmonology. Age of patients varied from 27 to 40 . Complete lower palsy was observed in 5 patients, and deep lowers paresis-in 1 patient. Palsy of the thoracic region of the spine or spinal cord was noted in 4 patients, and of the thoracic-lumber region- in 1 patient. Severity of the disease was evaluated as type A according to Frankel scale-in 4 patients, type B-in 1 patient and type $\mathrm{C}$-also in 1 patient.

Remoteness of the disease varied from 1.5 to 9 years. High muscle tone was examined in 3 patients, reduced tone-also in 3 patients. All patients were not able to stand and walk neither independently nor with the help of the corresponding person. Investigations have been carried out by means of the force measure insoles Tekscan. These insoles were put in the footwear of patients. Recording of the ground reaction force has been carried out during walking of patients in the exoskeleton before and after the course of training. Duration of the course comprised 2 weeks. The following parameters of walking have been calculated from the received data: main parameters (velocity, 
cadence and double step length), temporal indices (duration of the stance, swing and double support phases, rhythm coefficient), and dynamic parameters of walking-vertical component of the ground reaction force (GRF), phases of heel-strike, push-off and minimum) and distribution of pressure under the foot. The mean values of all of the above mentioned parameters of walking have been calculated with the account of their displacement during the locomotor cycle and graphs of the vertical component Rz of the ground reaction force have been drawn.

\section{Results}

Walking velocity in the exoskeleton in 5 healthy trained people in the exoskeleton comprised $0.79 \mathrm{~km} / \mathrm{hour}$, double step length $-0.66 \mathrm{~m}$, cadence-40 steps/min. During walking with such velocity duration of the stance phase is equal to $70 \%$, and duration of the swing phase comprises $30 \%$, for all this duration of the double-support phase grows to $20 \%$ in both legs. Increase of duration of the double -support phase in healthy people during walking in the exoskeleton is connected, firstly, with growth of duration of the locomotor cycle and, secondly, with application of crutches, because transfer and support on them demands the additional time, especially in the beginning of training. Transformation of the dynamic parameters of walking, namely of the vertical component Rz of the ground reaction force has been of our main interest. The vertical component in both legs has the traditional two-peaked form. But heel-strike is realized at the level of $27 \%$ of the locomotor cycle, then very long transfer to the minimal value takes place, its extreme value is timed to $39 \%$ of the locomotor cycle, followed by very short phase of push-off, which is realized at the level of $58 \%$ of the locomotor cycle. For all this the extreme values of Rz curve in both legs don't achieve the level of the body's weight: value of heel-strike is equal to $94 \%$, of the push-off- $94 \%$, of the minimum- $84 \%$. During walking of healthy trained people in the exoskeleton, similar to usual walking without exoskeleton, minimum of the vertical component $\mathrm{Rz}$ appears in the single-support phase, i.e. during the swing phase of the contra lateral leg. The main parameters of walking in 6 patients with paraplegia are inconsiderably reduced during walking in the exoskeleton in comparison with healthy examinees: double step length is diminished by $9 \%(0.6 \mathrm{~m})$, cadenceby $10 \%$ (36 steps $/ \mathrm{min}$ ) and mean walking velocity - by $18 \%(0.65$ $\mathrm{km} /$ hour). Duration of the stance phase comprises $80 \%$ of the cycle, and duration of the swing phase is equal to $20 \%$. At the same time duration of the double support phase is equal to $30 \%$, i.e. it is higher in comparison with the healthy people by $50 \%$.

But the dynamic parameters of walking change to the greatest extent, in particular, the vertical component $\mathrm{Rz}$ of the ground reaction force. Mean data of the vertical component of the ground reaction force in norm and in group of patients with spinal cord injury are presented in Figure 1. As may be seen from this figure, the vertical component of the ground reaction force acquires the trapezium form. All of the extreme values are remarkably reduced in comparison with norm in both legs and don't exceed the level of the body's weight. Clear asymmetry of the dynamic parameters is observed. Considerable displacement of all of the extreme values to the right along the temporal axis is noted. The heel strike (value $-49 \%$ in the left leg and $76 \%$ - in the right leg) is developed only by $\mathrm{t}=38 \%$ of the locomotor cycle, then slow transfer to the minimum appears, at the level of $62 \%$ of the cycle (values are equal correspondingly to $30 \%$ and $41 \%$ ), and at last very rapid development of the push-off takes placeat the level of $77 \%$ of the cycle (values are equal to $54 \%$ and $78 \%$ ). It is believed, that at the first stage of mastering the skill of walking in the exoskeleton patients must increase duration of the heel-strike for providing the static stability. Remarkable increase of all extreme values of ground reaction force is noted. In comparison with the initial data, value of the heel-strike in the left leg grows in $62 \%(80 \%)$, of the minimum-in $85 \%$ (55\%), of the push-off-in $46 \%$ (75\%). Lesser growth of amplitude of the vertical components is observed in the right leg in comparison with the left one, correspondingly value of the heel-strike and push-off increase in $14-17 \%$ in comparison with the initial data (correspondingly $-87 \%$ and $86 \%$ ), and of the minimum-in $61 \%(65 \%)$. The real data of GRF, recorded by means of the force sensor, during walking of patient with complete lower palsy are given in Figure 2.

At the beginning of the course the value of heel strike in the left leg comprised $51 \%$ in the left leg and $62 \%$ in the right leg. The form of the vertical component of both legs points to the unstable position of the foot on the support surface, what is revealed in presence of a lot of small peaks in both curves in fig. A. After the course of training in the exoskeleton values of the vertical component of the ground reaction force remarkably increase, especially value of heel strike up to $89 \%$ in the left leg and up to $101 \%$ in the right leg. For all this the minimal value is displaced to the left along the temporal axis. After the course the form of the curves becomes more smooth, small peaks disappear, minimal values are displaced to the left along the temporal axis. Values of the push-off also increase in both legs - from 30\% up to $70 \%$ in the left leg and from $42 \%$ up to $84 \%$ in the right leg, what testifies to mastering the skill of walking in the exoskeleton.
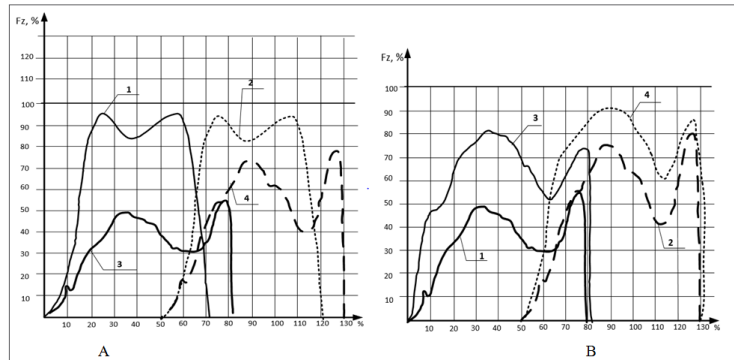

Figure I Vertical component $\mathrm{Rz}$ of the ground reaction force (GRF) during walking in the exoskeleton in patients with consequences of the spinal cord injury before and after the course of rehabilitation and the healthy examinees. Figure A-thin solid line I and thin dotted line 2-Rz components of GRF of the left I and right 2 legs in the healthy examinees; thick solid line 3 and thick dotted line $4-\mathrm{Rz}$ components of the left 3 and right 4 legs during walking of patients with consequences of the spinal cord injuries. Figure $\mathrm{B}-$ Vertical component $\mathrm{Rz}$ of the GRF during walking in the exoskeleton in patients with consequences of spinal cord injury before and after the course of rehabilitation. I and 2-Rz component of GRF before the course, 3 and 4-Rz component of GRF after the course. I and 3-left leg, 2 and 4-right leg.

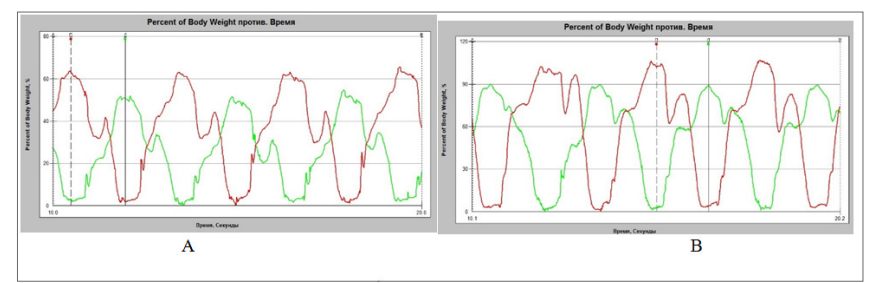

Figure 2 Example of the vertical component of the ground reaction force during walking of patient with consequences of the spinal cord injury. Avertical component $\mathrm{Rz}$ of a ground reaction force during walking in the exoskeleton before the course, B-vertical component Rz of the same patient after the course. Red color-Rz curve of the right leg, green color-Rz curve of the left leg. 


\section{Conclusion}

Force measure insoles make it possible to receive the following informative parameters of walking: main indices of the gait, phase structure of the locomotor cycle, the extreme values of the ground reaction force in the locomotor cycle. Application of the force measure insoles permits to investigate change of stability and degree of adaptation of patient to walking in the exoskeleton, and also to retrace the results of complex rehabilitation of patients with complete lower palsy. The further investigations in this field will be directed to investigation the possibility of application of this method in the other diseases of the locomotor system.

\section{Acknowledgements}

None.

\section{Conflict of interest statement}

The author declares that the research was conducted in the absence of any commercial or financial relationships that could be construed as a potential conflict of interes

\section{References}

1. Miller LE, Zimmermann AK, Herbert WG. Clinical effectiveness and safety of powered exoskeleton-assisted walking in patients with spinal cord injury: systematic review with meta-analysis. Med Devices (Auckl). 2016;22(9):455-66.

2. Fisahn C, Aach M, Jansen O, et al. The effectiveness and safety of exoskeletons as assistive and rehabilitation device in the treatment of neurologic gait disorders in patients with spinal cord injury: a systematic review. Clobal Spine J. 2016;6(8):822-841.

3. Sylos-Labini F, La Scaleia V, D Avella A, et al. EMG patterns during assisted walking in the exoskeleton. Front Hum Neurosci. 2014;16(8):423.

4. Del-Ama AJ, Koutsou AD, Moreno JC, et al. Review of hybrid exoskeleton to restore gait following spinal cord injury. Journal of Rehabilitation Research and Development. 2012;49(4):497-514.

5. Esquenazi A, Talaty M, Packel A, et al. The ReWalk powered exoskeleton to restore ambulatory function to individual with thoracic-level motorcomplete spinal cord injury. Am J Phys Med Rehabil. 2012;91(11):911-21.

6. Hartigan C, Kandilakis C, Dalley S, et al. Mobility outcomes, following five training sessions with a powered exoskeleton. Top Spinal Cord Injury Rehabilitation. 2015;21(2):93-9.

7. Kozlowski AJ, Bryce TN, Dijkers MP. Time and Effort, required by persons with spinal cord injury to learn to use a Powered Exoskeleton for assisted walking. Spinal CordInjury Reabilitation. 2015;21(2):110-21.

8. Raab K, Krakow K, Tripp F, et al. Effects of training with the ReWalk exoskeleton on quality of life in incomplete spinal cord injury: a single case study. Spinal Cord Series and Cases. 2016;3:15025.

9. Vitenson AS, Petrushanskaja KA. From natural to artificial management of locomotion (From the natural then the artificial control of locomotion). M, Scienific medical firms. MBN. 2003:448.

10. Fineberg DB, Asselin P, Harel NY, et al. Vertical ground reaction forcebased analysis of powered exoskeleton-assisted walking in persons with motor-complete paraplegia. The Journal of Spinal Cord Medicine. 2013;36(4):313-21. 